\title{
Disrupted white matter in language and motor tracts in developmental stuttering
}

Article

Accepted Version

Connally, E., Ward, D., Howell, P. and Watkins, K. E. (2013)

Disrupted white matter in language and motor tracts in developmental stuttering. Brain and Language, 131. pp. 25-35. ISSN 0093-934X doi:

https://doi.org/10.1016/j.bandl.2013.05.013 Available at https://centaur.reading.ac.uk/31405/

It is advisable to refer to the publisher's version if you intend to cite from the work. See Guidance on citing.

To link to this article DOI: http://dx.doi.org/10.1016/j.bandl.2013.05.013

Publisher: Elsevier

All outputs in CentAUR are protected by Intellectual Property Rights law, including copyright law. Copyright and IPR is retained by the creators or other copyright holders. Terms and conditions for use of this material are defined in the End User Agreement.

\section{www.reading.ac.uk/centaur}

\section{CentAUR}

Central Archive at the University of Reading 
Reading's research outputs online 


\section{Disrupted white matter in language and motor tracts in developmental stuttering}

Emily L. Connally ${ }^{1}$, David Ward ${ }^{2}$, Peter Howell ${ }^{3}$, Kate E. Watkins ${ }^{1}$

${ }^{1}$ Department of Experimental Psychology \& Oxford Centre for Functional MRI of the Brain (FMRIB), University of Oxford, South Park Road, Oxford, OX1 3UD, UK ${ }^{2}$ School of Psychology and Clinical Language Sciences, University of Reading, Earley Gate, Whiteknights Road, Reading, RG6 6AL, UK

${ }^{3}$ Cognitive, Perceptual and Brain Sciences Department, University College London, 26 Bedford Way, London, WC1H 0AP UK

Email:

connally@fmrib.ox.ac.uk

d.ward@reading.ac.uk

p.howell@ucl.ac.uk

kate.watkins@psy.ox.ac.uk

Corresponding author:

Emily Connally

Department of Experimental Psychology

University of Oxford

South Parks Road

Oxford, OX1 3UD

UK

Tel: +44 1865271444

Fax: +44 1865319447 


\begin{abstract}
White matter tracts connecting areas involved in speech and motor control were examined using diffusion-tensor imaging in a sample of people who stutter $(n=29)$ who were heterogeneous with respect to age, sex, handedness and stuttering severity. The goals were to replicate previous findings in developmental stuttering and to extend our knowledge by evaluating the relationship between white matter differences in people who stutter and factors such as age, sex, handedness and stuttering severity. We replicated previous findings that showed reduced integrity in white matter underlying ventral premotor cortex, cerebral peduncles and posterior corpus callosum in people who stutter relative to controls. Tractography analysis additionally revealed significantly reduced white matter integrity in the arcuate fasciculus bilaterally and the left corticospinal tract and significantly reduced connectivity within the left corticobulbar tract in people who stutter. Region-of-interest analyses revealed reduced white matter integrity in people who stutter in the three pairs of cerebellar peduncles that carry the afferent and efferent fibers of the cerebellum. Within the group of people who stutter, the higher the stuttering severity index, the lower the white matter integrity in the left angular gyrus, but the greater the white matter connectivity in the left corticobulbar tract. Also, in people who stutter, handedness and age predicted the integrity of the corticospinal tract and peduncles, respectively. Further studies are needed to determine which of these white matter differences relate to the neural basis of stuttering and which reflect experience-dependent plasticity.
\end{abstract}

\title{
Keywords
}

fluency disorder, speech production, structural image analysis, fiber tracking, arcuate fasciculus, posterior limb of internal capsule 


\section{Introduction}

Persistent developmental stuttering arises in early childhood, remits before puberty in around $80 \%$ of cases, and persists to adulthood in $1 \%$ of the population (Andrews \& Harris, 1964). Developmental stuttering is a disorder of speech fluency marked by involuntary sound repetition and prolongation, articulation cessation (blocks), and sometimes accompanied by marked oromotor disturbance likened to facial tics in disorders of motor control (e.g. Tourette's syndrome) (Ludlow \& Loucks, 2003; Yairi \& Ambrose, 1999). Though the symptoms of stuttering can sometimes be quite severe, there are usually no associated brain lesions, neuropathology, or impairment in other areas of cognition. As such, people who stutter (PWS) comprise a unique population with focal dysfluency for exploring the neural underpinnings of speech production. Furthermore, the high rate of recovery in PWS during childhood (Månsson, 2000) offers an opportunity to investigate mechanisms underlying neuronal plasticity and spontaneous recovery.

With the availability of non-invasive brain-imaging methods, the number of studies of brain structure and function in PWS has rapidly increased. Even so, the precise neural basis of stuttering remains elusive and its ontogenesis is still poorly understood. Hypotheses regarding incomplete cerebral dominance (Travis, 1978), altered basal ganglia activity (e.g. Alm, 2004), and abnormalities in predicting the sensory consequences of motor speech (e.g. Neilson \& Neilson, 1987; Max, Guenther, Gracco, Ghosh, \& Wallace, 2004) have all been considered as explanations of stuttering. Support from functional imaging studies for these theories is mixed, however. For example, a meta-analysis of eight early functional imaging studies (Brown, Ingham, Ingham, Laird, \& Fox, 2005) described right hemisphere over-activity in PWS, which is consistent with altered cerebral dominance in this population. The same analysis found an "absence" of activity in auditory cortex bilaterally and overactivity in the cerebellum, lending support to the idea that sensorimotor integration is abnormal in stuttering (De Nil, Kroll, Kapur, \& Houle, 2000). The meta-analysis did not reveal abnormal function in the basal ganglia of PWS, however. The numerous functional imaging studies published since 2005 have not helped to clarify the picture, replicating in part previous findings but on the whole failing 
to identify a clear set of functional correlates of stuttering (e.g. Chang, Kenney, Loucks, \& Ludlow, 2009; Giraud et al., 2008; Loucks, Kraft, Choo, Sharma, \& Ambrose, 2011). Studies of functional connectivity during task and at rest show promise in studies of stuttering (Chang, Horwitz, Ostuni, Reynolds, \& Ludlow, 2011; Lu et al., 2010; Xuan et al., 2012) and could be sensitive to changes in speech networks due to treatment and recovery (e.g. Lu et al., 2012).

Analysis of brain structure in PWS has also produced varied results: reduced cerebral asymmetry and altered sulcal anatomy have been reported but not replicated (e.g. Mock et al., 2012;Chang, Erickson, Ambrose, HasegawaJohnson, \& Ludlow, 2008; Cykowski et al., 2007; Foundas et al., 2003; Foundas, Bollich, Corey, Hurley, \& Heilman, 2001). Similarly, grey matter is described as increased in several brain areas in adults who stutter relative to controls (Beal, Gracco, Lafaille, \& De Nil, 2007; Cykowski et al., 2007; Jancke, Hanggi, \& Steinmetz, 2004) but decreased in children who stutter (Chang et al., 2008). Also, the volume of the rostral half of the corpus callosum was assessed to be increased in adults who stutter relative to controls (Choo et al., 2011) but there were no differences among groups of boys who persisted in or recovered from stuttering and those who were normally fluent (Choo, Chang, Zengin-Bolatkale, Ambrose, \& Loucks, 2012).

The lack of a consensus picture across functional imaging studies may relate to the sensitivity of these methods to functional differences in PWS that are due to differences in the speech act itself (including, but not only, dysfluent speech). Structural image analysis is uninfluenced by these dynamic differences but both methodologies are also sensitive to differences in PWS that reflect the etiology (possibly genetic) of the disorder as well as the brain's plastic response to it. A major challenge in stuttering research is sifting through these findings in an attempt to determine what sorts of anomalies indicate plasticity and which are more likely to reflect congenital disorganization or disconnection.

Against this background of varied findings of differences in brain function and structure in PWS is the small set of studies that show an abnormality affecting the white matter underlying left ventral sensorimotor cortex (e.g. (Chang et al., 2008;Cykowski et al., 2010; Kell et al., 2009; Sommer, Koch, Paulus, Weiller, \& Büchel, 2002; Watkins, Smith, Davis, \& Howell, 2008). These reports 
used diffusion-weighted imaging to measure white matter integrity in PWS. The microstructure of white matter is quantitatively assessed using fractional anisotropy (FA), a ratio of diffusion of water along the principle diffusion direction (longitudinal diffusivity, L1) to that along the two orthogonal axes (radial diffusivity, RD). Barriers to diffusion, such as the structure of white matter fiber tracts and their layers of myelin, impede radial diffusivity (Beaulieu, 2009). The principal diffusion direction is often used, therefore, to infer the trajectory of large well-aligned fiber bundles and FA is used to measure white matter integrity or organization. Low FA might reflect disorganization of white matter tracts, because longitudinal diffusivity is reduced, for example in areas of crossing fibers, or because radial diffusivity is increased, perhaps due to myelin disruptions. In PWS, reduced FA in white matter was associated with increased radial diffusivity (Cykowski et al., 2010), which can be interpreted as indicative of fewer barriers to diffusion across the fiber tracts. An increase in radial diffusivity in PWS possibly reflects abnormal myelin development.

The finding of reduced FA in the white matter underlying left ventral sensorimotor cortex of PWS across several studies is reassuring, particularly as the studies differed in the number of diffusion directions acquired, analysis approaches and in the demographics of the populations studied. The effect is especially interesting given that PWS showed decreased activity during speech production (in functional MRI) in the same region they showed reduced FA (Watkins et al., 2008). It should be noted, however, that at least one report found elevated FA in white matter close to this region in PWS (Kell et al., 2009).

Several other questions regarding the nature of the white matter abnormalities in stuttering remain. Not least of these, is whether structural differences reflect a possible cause of the disorder, perhaps due to genetic disruptions, or are a consequence of life-long stuttering behaviour. Longitudinal analyses are required to address this question, but the persistence of the abnormality in children who recovered from stuttering (Chang et al., 2008) would seem to rule out the idea that this particular white matter difference reflects experience-dependent plastic change in response to stuttered speech. Other questions relate to the relationships between white matter differences and sex, handedness, age and stuttering severity in PWS. Sex and handedness have 
been shown to influence sulcal anatomy and grey matter volume in language areas (Powell, Kemp, Roberts, \& García-Finaña, 2012) in healthy controls, but the degree to which stuttering might impact the influence of those factors is still undetermined. The factors of sex and handedness are often considered to be confounds contributing to the lack of clear structural and functional correlates of stuttering. Consequently, studies have aimed to address this by focussing analyses on the largest homogeneous subpopulation of stutterers, usually righthanded males who stutter (e.g. Cykowski et al., 2007, 2010).

One of the aims of the current study was to report results from a large and heterogeneous sample of PWS and examine separately how the factors contributing to this heterogeneity affect the white matter differences detected. We considered age, sex, handedness and stuttering severity in these analyses. We also used probabilistic tractography and region-of-interest analyses to focus on specific pathways involved in speech and motor control.

\section{Methodology}

\subsection{Participants}

Twenty-nine PWS (mean age 22.6 years; range 14 to 42 years; eight females; 21 males) and 37 fluent controls (CON; mean age 24.3 years; range 14 to 45 years; 14 females; 23 males) were scanned using diffusion-weighted imaging. The groups were well matched for age and proportion of males to females. Three of the 21 males and four of the eight female PWS were left-handed; there were two left-handed males in the CON group. The PWS group ranged in stuttering severity, as assessed by two independent raters using the Stuttering Severity Instrument-3, SSI-3, (Riley, 1994) from mild to severe (mean 22.9; range 8 to 38.5). An SSI-3 score was not available for one right-handed male participant whose stuttering was estimated as mild relative to those in the cohort. The data from some of the group were used in a previous report (Watkins et al., 2008) but further data have been added from both PWS and fluent-speaking controls.

\subsection{Imaging}


Structural and diffusion-weighted MRI images of the whole brain were acquired using a 1.5T Siemens Sonata clinical imaging system. High-resolution T1weighted images (1-mm isotropic voxels, FLASH sequence, TR=12ms, TE=5.65 $m s$, flip angle $=19^{\circ}$ ) were obtained in each participant and coregistered to the MNI152 template. Two sets of echo-planar images were acquired (53 x $2.5 \mathrm{~mm}$ axial slices, in-plane resolution $2.5 \mathrm{~mm}^{2}$ ). Each set included three non-diffusionweighted and 60 diffusion-weighted images acquired with a $b$-value of 1000 $\mathrm{smm}^{-2}$ uniformly distributed across 60 gradient directions. Diffusion data were preprocessed using the FMRIB Diffusion Toolbox (FDT v2.0; http://www.fmrib.ox.ac.uk/fsl;Behrens et al., 2003; Behrens, Johansen-Berg, Jbabdi, Rushworth, \& Woolrich, 2007). Images were corrected for eddy currents and head motion by affine registration to non-diffusion volumes. Data were averaged across the two sets to improve signal to noise ratio. Images of FA, longitudinal (or axial) diffusivity along the primary diffusion direction (L1) and average radial diffusivity (RD) across the two axes perpendicular to L1 were created. Diffusion images were registered, using boundary-based registration (Greve \& Fischl, 2009), first to each participant's T1-weighted image and then to the MNI152 standard space brain for group comparisons. Probabilistic tractography was run using the BedpostX and ProbtrackX functions in FDT.

\subsection{Analysis}

\subsubsection{Cerebral White Matter}

Tract-Based Spatial Statistics. Voxel-wise statistical analysis of the FA data was performed using Tract-Based Spatial Statistics (TBSS, Smith et al., 2006). TBSS registers the diffusion images non-linearly to an in-house target image obtained from a high-resolution average of 58 healthy participants aligned to standard space. The average of all data was reduced to a 1-mm thick white matter skeleton that should align with the center of white matter tracts common across participants. For each participant's non-linearly registered FA image, the highest value of FA nearest to the skeleton was projected onto the skeleton for analysis. A t-test was performed at each voxel in the skeleton. Permutation testing was performed using the Randomise function in FSL (10,000 permutations) and statistical inference was based on an uncorrected voxel-based threshold $\mathrm{p}<0.005$ 
with an extent threshold of at least 10 voxels (there were no significant differences in FA between PWS and CON when fully corrected for multiple comparisons). FA was compared between the groups of PWS and CON while the effects of the confound regressors age, sex, and handedness were accounted for through inclusion as covariates in the model. Significant group differences in FA were further explored by analysis of the $\mathrm{L} 1$ and RD values that contribute to the calculation of FA; planned group comparisons of these values are reported at p $<0.05$ uncorrected. The correlation between FA and SSI-3 was also tested in regions showing significant group differences. Again, these exploratory analyses are reported at $\mathrm{p}<0.05$ uncorrected.

Tractography. Probabilistic tractography was used to identify the corticospinal tract (CST) and arcuate fasciculus (AF) in each hemisphere and for each participant. This analysis used previously described methods for defining seed and target masks in standard space (Giorgio et al., 2010). For the AF, a seed mask was placed in a single coronal slice $(y=-38)$ at the arc of the tract and two target masks were placed along that tract, one coronal mask in the white matter anterior to the seed mask, and another axial mask ventral to the seed mask. Exclusion masks including one for the midline were used to remove any branches extending outside of the AF and termination masks were located immediately outside the target masks. The pathways resulting from the tractography were thresholded in each participant in order to isolate the $\mathrm{AF}$ more confidently; only voxels through which at least 500 (out of 5000 per voxel of the seed and target masks) samples passed were retained. See Figure 2A for placement of AF masks and resulting tract. The CST seed mask was located in the pons on an axial slice at $z=-22$ with an axial target mask in dorsal white matter of the same hemisphere underlying the motor cortex at the level of $z=26$.

Termination masks included the area superior to and surrounding the target mask and the whole brain inferior to the seed mask. An exclusion mask ran through the midline. The pathways resulting from the tractography were thresholded in each participant to remove spurious connections; only voxels through which at least 20 samples passed were retained (Giorgio et al., 2010). See Figure 2B for placement of CST masks and resulting tracts. 
The CST tracked as above comprises mostly fibers from the dorsal corona radiata travelling in the middle part of the posterior limb of the internal capsule to the pons. For comparison, the corticobulbar tract (CBT) fibers were isolated so that the hypothesis that they are selectively involved in the pathology underlying stuttering could be tested. Probabilistic tractography between the seed mask placed in the pons (axial slice at $\mathrm{z}=-22$ as above) and two new target masks placed in the most dorsal aspect of the internal capsule were used. The two target masks comprised 9 voxels each (3x3 square) and they were drawn in the posterior limb of the internal capsule on each participant's FA modulated primary diffusion direction images (first eigenvector), in which the orientation of fibers can be inferred from the color (red for left-right, blue for superior-inferior and green for anterior-posterior). The targets were drawn in the same axial slice, identified as a cross-section through the basal ganglia in which both genu and splenium of the corpus callosum were visible and the internal capsule was at its broadest (Bonekamp et al., 2007). The new target that was used to isolate the CBT was located close to the genu of the posterior limb of the internal capsule comprising mostly fibers with a superior-inferior orientation (blue color). The target used to isolate the CST was positioned just posterior and lateral to the CBT target in the middle portion of the posterior limb of the internal capsule (also comprising fibers with a superior-inferior orientation). Termination masks covered the brain tissue ventral to the seed mask in the pons (as for CST above) and the whole axial slice above the two target masks (see Figure 3). Tracking the CBT from cortex to pons would require traversing the fibers orientated rostrocaudally that comprise the superior longitudinal fasciculus (SLF). These crossing-fibers may confound measurements made in the CBT. By restricting the analysis to the ventral portion of the tracts, we avoided the potential confound introduced by tracking across the SLF. The pathways resulting from this tractography were thresholded at a minimum of 20 samples (as above).

Total volume, mean FA, and the number of streamlines (total number of tracts generated from the seed mask that reached the target masks) were measured for each probabilistic tract. Data were compared using analysis of variance (ANOVA), with a between-subjects factor of group (PWS vs. CON) and a within-subjects factor of hemisphere (left vs. right). For the tract data in the 
posterior limb of the internal capsule, another within-subjects factor of tract (CBT vs. CST) was added. The contribution of age, sex, handedness and stuttering severity (SSI-3 score) to the measures that showed significant differences from CON in PWS were evaluated using step-wise linear regression.

\subsubsection{Cerebellar White Matter}

The cerebellum was excluded from the TBSS analysis partly because the cerebellar peduncles are slender and not always robustly detected by the TBSS skeleton (presumably due to differences in registration). Also, it is not possible to dissociate specific tracts within the cerebellar white matter using the skeleton approach in TBSS. Instead, the integrity of cerebellar white matter was evaluated using ROIs for the peduncles, and the segmented white matter of each lobe and vermis.

Cerebellar lobes. Within the cerebellum, mean FA was calculated for the white matter in five ROIs: the vermis and the anterior and posterior lobes, bilaterally. These ROIs were drawn on the T1-weighted structural images in MNI152 standard space. The posterior vermis was identified using probability maps (thresholded at 20\%) previously published (Diedrichsen, 2006) and included as an atlas in the FSLView software. The anatomical boundaries for the anterior lobe vermis are unclear; therefore, data from this region were excluded by removing voxels in the midsagittal plane $(x=0)$ for the anterior lobe ROIs. The four lobes were drawn using the midline and primary fissure as boundaries between hemispheres and anterior and posterior ROIs, respectively, and once drawn the vermis mask was subtracted from the masks of the lobes to yield five masks with no overlapping voxels. Only the posterior vermis ROI contained voxels in the midline. These five ROIs were transformed from standard space to the participant's structural image. White matter voxels in these ROIs were identified using the partial volume estimate for white matter, which was the produced by the segmentation procedure used during the boundary-based registration to standard space. Voxels that had a partial volume estimate of $>$ 0.99 (i.e. were completely white matter) were retained and the mean FA across these voxels for each ROI was calculated. Group differences in FA were assessed 
using a mixed ANOVA with between-subject factor of group (PWS vs. CON) and a within-subject factor of ROI (five: left and right anterior and posterior lobes and vermis).

Cerebellar peduncles. In order to better isolate the afferent and efferent pathways of the cerebellum, one ROI was placed in each of the cerebellar peduncles of each participant, bilaterally, for a total of six ROIs per participant. Masks were drawn using FA modulated primary diffusion direction images (color coded) to identify regions in the superior (SCP, 10 voxels), middle (MCP, 18 voxels) and inferior (ICP, 8 voxels) connections between the brainstem and the cerebellum (see Klein et al., 2011 for details). In essence, this involved placing the ROIs in the center of these peduncles. The SCP is visible on sagittal slices close to the midline as the more dorsal connection from the posterior margin of the brainstem to the cerebellum travelling in an anterior-posterior/superior-inferior direction. The SCP ROI comprised 10 voxels in the middle of the peduncle in up to three contiguous sagittal slices. The MCP is the largest peduncle and clearly identified on an axial slice at about the level of the mid pons as large tracts extending in the anterior-posterior direction bilaterally with obvious laterally-crossing pontine fibers between those extensions anteriorly. ROIs were drawn on two contiguous coronal slices ( 9 voxels each) located immediately posterior to the ICP, which is visible ventral to the MCP on coronal slices. ICP ROIs were always inferior to the SCP, anterior to the MCP, and were identified on a single sagittal slice as steep inferior-superior extensions. ICP ROIs were drawn on two contiguous coronal slices ( 4 voxels each) such that the entire ROI could be seen in a single sagittal slice. See Figure 4 for placement of cerebellar peduncle ROIs in a representative subject. FA was extracted from these images and subjected to ANOVA with the between-subject factor of group (PWS vs. CON) and two within-subject factors of peduncle (SCP vs. MCP vs. ICP) and hemisphere (left vs. right). Significant differences in FA involving group were further explored for these small ROIs by analysis of the underlying L1 and RD values that contribute to FA. Cerebellar white matter measures that showed significant group differences explored further with step-wise linear regression to evaluate their relationships with age, sex, handedness and stuttering severity (SSI-3 score) in PWS. 


\section{Results}

\subsection{Cerebral White Matter}

\subsubsection{Tract-based spatial statistics}

The PWS and CON groups did not differ significantly in mean FA across all cerebral voxels and all voxels in the skeleton (cerebral FA: CON $0.225 \pm 0.008$, PWS $0.222 \pm 0.009$; skeletal FA: CON $0.438 \pm 0.015$, PWS $0.434 \pm 0.016$ ). There were no significant group differences in the TBSS analyses following correction for multiple comparisons using threshold-free cluster enhancement. Therefore, group differences in FA are reported at an uncorrected threshold of $p<0.005$ when they extended for at least 10 contiguous voxels. Differences that survived the $p<0.005$ threshold that comprised fewer than 10 voxels are also reported if they were in areas contralateral to a homologous region in the other hemisphere that survived the extent threshold of $>10$ voxels. At this uncorrected threshold, several regions showed significant differences between the two groups and many occurred bilaterally (the significance of these pairs is $\mathrm{p}<0.005^{2}$; see Table 1). PWS had significantly lower FA than CON: (i) bilaterally in white matter underlying the superior frontal gyrus, anterior inferior frontal gyrus, ventral premotor cortex, angular gyrus, lateral occipital cortex, forceps major, optic radiations and the cerebral peduncles; (ii) in the body of the corpus callosum; (iii) in multiple areas in the left hemisphere including the white matter underlying the middle frontal gyrus and posterior middle temporal gyrus, the anterior corona radiata and superior longitudinal fasciculus; and (iv) in the right ventral temporal and occipital white matter (see Figure 1). PWS had significantly higher FA than CON in the white matter underlying the middle frontal gyrus and dorsal precentral gyrus bilaterally and the left temporal pole.

Figure 1 about here

$\mathrm{RD}$ and $\mathrm{L} 1$ were examined in order to understand better the microstructure underlying FA differences. Across all these areas, when the PWS group had lower FA than CON, they also had lower diffusivity along the longitudinal axis of the tract (L1), whereas in those regions where PWS had higher FA than CON, they also had higher L1. RD averaged across the two directions orthogonal to the principal diffusion direction was significantly higher 
in PWS than CON in the following regions in which FA was lower in PWS than CON: angular gyrus and optic radiations bilaterally, the body of the corpus callosum, the left cerebral peduncle, right superior frontal gyrus and fusiform gyrus (see Table 1). RD was significantly lower in PWS than CON in the following regions in which FA was higher in PWS than CON: dorsal precentral gyrus bilaterally and right middle frontal gyrus (see Table 1).

Table 1 about here

Correlations between FA and stuttering severity were explored within the PWS group. FA in the left angular gyrus was lowest in participants with the highest SSI-3 (negative correlation; $r=-0.41, p=0.03$; Figure 5A).

\subsubsection{Tractography}

The arcuate fasciculus (AF) and corticospinal tract (CST) were successfully tracked bilaterally in all participants using probabilistic tractography. The mean FA for the total tract, the tract volume and the number of streamlines in the PWS and CON groups were compared for these anatomically-defined tracts using ANOVA.

For the AF, PWS had significantly lower FA relative to the CON group across both hemispheres $(F(1,64)=5.04, p=0.028)$ (Figure $2 \mathrm{~A})$. The PWS and CON groups did not differ in volume of the SLF or in the number of streamlines. There was a significantly higher number of streamlines in the left compared to the right hemisphere across both groups $(F(1,64)=8.09, p=0.006)$. Age, sex, handedness and SSI-3 score did not significantly predict FA in the left or right SLF or the average FA across the two sides in PWS.

For the CST, PWS had lower FA on the left compared with the right $(t(28)=4.18, p<0.001)$, whereas there was no difference in FA between hemispheres in CON (group x hemisphere interaction: $F(1,64)=7.06, p=0.010$; see Figure 2B). PWS and CON groups did not differ in the volume of the CST or in the number of streamlines. Age, sex, handedness and SSI-3 score did not significantly predict FA in the left or right CST separately in PWS. Handedness, however, was a significant predictor of the difference in FA between the two hemispheres in PWS (model fit: $F(1,26)=4.52, p=0.043 ; r=0.39, p=0.022$ ). In right-handers who stutter, FA tends to be higher in the right CST than the left, 
whereas in the small number of left-handers who stutter the average difference between FA in the two hemispheres was negligible (see Figure 5B).

Figure 2 about here

\subsubsection{Posterior Limb of Internal Capsule.}

The CBT and CST from the posterior limb of the internal capsule to the cerebral peduncles were successfully tracked bilaterally using probabilistic tractography in all participants. For these tracts, the mean FA for the total tract, tract volume and number of streamlines were compared between the PWS and CON groups.

Analysis of the mean FA of these tracts revealed that there were hemispheric differences that were different for the two tracts in the two groups (group $\mathrm{x}$ tract $\mathrm{x}$ hemisphere interaction: $F(1,64)=5.00, p=0.029$ ). Separate analysis for the left and right hemispheres revealed that this interaction was due to higher FA in PWS relative to CON in the right CBT with no differences in the right CST (group x tract interaction: $F(1,61)=4.13, p=0.046$ ) and no group differences in the left CBT and CST. Age, sex, handedness and SSI-3 score did not significantly predict FA in the right CBT in PWS.

A similar pattern was seen for the analysis of tract volume (significant group $\mathrm{x}$ tract $\mathrm{x}$ hemisphere interaction: $F(1,64)=6.23, p=0.015)$. This interaction was due to a significant reduction in volume of the CST in PWS relative to CON in the left hemisphere $(t(64)=2.24, p=0.029)$ but not the right; the left CBT volume in PWS was also reduced but this difference was not quite significant $(t(64)=1.87, p=0.066)$. Age, sex, handedness and SSI-3 score did not significantly predict the volume of the left CST in PWS.

There were significantly fewer streamlines in PWS relative to CON in the left hemisphere in both the CBT and the CST (group $\mathrm{x}$ hemisphere interaction: $F(1,64)=4.678, p=0.034)$. In the PWS group, there were significantly fewer streamlines in the left compared with the right CBT $(p=0.001)$, but the left and right CST did not differ; there was no difference between hemispheres for either tract in the CON group (see Figure 3). SSI-3 score was the only significant predictor of the number of streamlines in the left CBT in PWS (model fit: $F(1,26)=5.34, p=0.029 ; r=0.41, p=0.015)$; age, sex and handedness did not 
improve the model fit. This positive relationship indicates that the number of streamlines in the left CBT is highest in those who have the highest SSI-3. As the direction of this relationship was unexpected, we further explored whether SSI-3 score related to the number of streamlines in the right CBT (this was also a positive relationship and close to significance, $p=0.089$ ) or the difference in the number of streamlines between the two hemispheres $(p=0.9)$.

Figure 3 about here

\subsection{Cerebellar White Matter}

\subsubsection{Cerebellar lobes}

FA was compared between groups for the white matter of the left and right, anterior and posterior cerebellar lobes and the vermis. Mean FA did not differ between PWS and CON groups in any of these areas.

\subsubsection{Cerebellar Peduncles}

PWS had significantly lower FA than CON in each pair of cerebellar peduncles $(F(1,64)=21.55, p<0.001$; see Figure 4$)$. For both groups, FA was significantly lower in the left peduncle compared to the right $(F(1,64)=7.97$, $p=0.006$ ) and in the inferior cerebellar peduncles relative to the middle cerebellar peduncles, which were, in turn, significantly lower than the superior cerebellar peduncles $(F(2,64)=100.6, p<0.001)$. This pattern did not differ between groups or hemispheres. There were no differences between PWS and CON groups in L1 and RD in any of the peduncles. Age was the only significant predictor of the average FA in the inferior cerebellar peduncles in PWS (model fit: $F(1,26)=9.31, p=0.005 ; r=0.51, p=0.003)$; sex, handedness and SSI- 3 did not improve the model fit. This positive relationship indicates that FA increases with increasing age.

Figure 4 and 5 about here

\section{Discussion}

Our objective was to use diffusion tensor imaging to examine for the neural correlates of developmental stuttering in the white matter tracts connecting speech and motor areas of the brain. The large sample studied comprised right- and left-handed male and female PWS and normally fluent 
controls who ranged in age from 14 to 42 years. The contributions made by these potential confounds to the white matter differences observed in PWS were examined. The main findings were that PWS have reduced white matter integrity (lower FA): (i) in a large number of areas throughout the cerebrum, often occurring in homologous regions in both hemispheres; (ii) in the arcuate fasciculus bilaterally; (iii) in the left corticospinal tract; and (iv) in the cerebellar peduncles. In addition, the connectivity of the left corticobulbar tract (as indexed by the number of streamlines) was significantly reduced in PWS. The more severe the dysfluency in the PWS group, the greater the reduction in white matter integrity in the left angular gyrus but the greater the connectivity in the corticobulbar tract. In PWS, right-handedness predicted a greater rightward asymmetry in the white matter integrity of the corticospinal tract and age predicted greater integrity in the inferior cerebellar peduncle.

\subsection{Relation to previous reports}

The current analysis using tract-based spatial statistics replicated corresponding findings from Watkins et al. (2008), which was based on data from the younger half (14-27 years) of the dataset reported here. It is not surprising, therefore, that decreased FA was found again bilaterally in the white matter underlying the ventral premotor cortex, an area that the previous study also found to be functionally hypoactive in the left hemisphere during speech production in PWS. As mentioned in the introduction, white matter abnormalities in the left ventral sensorimotor (peri-Sylvian or opercular) cortex have been described in a group of ten male and five female adult PWS (age range 18-44 years; one left-hander) (Sommer et al., 2002), in a group of 15 righthanded male children who persisted in or recovered from stuttering aged 9-12 years (Chang et al., 2008), and in a group of 13 right-handed male PWS (mean age 31 years; Cykowski et al., 2010). In contrast with this last study, however, we did not find any difference in radial diffusivity in this region in PWS.

Reduced white matter integrity (i.e. lower FA) in the region of the left ventral sensorimotor cortex could reflect disruption in one or more of several pathways in this area that are known to play a role in speech production. The largest candidate pathway in this region is the superior longitudinal fasciculus, 
which connects posterior temporal cortex, inferior parietal cortex and lateral prefrontal cortex (Catani, Howard, Pajevic, \& Jones, 2002; Petrides \& Pandya, 2009) and is important for integration of auditory and motor signals necessary for fluent speech. This tract contains fibres from the arcuate fasciculus (Catani, Jones, \& Ffytche, 2005; Petrides \& Pandya, 2002), traditionally "the language" pathway (Geschwind, 1975), lesions of which were thought to cause conduction aphasia (but see Hillis, 2007). This region also contains small, local, corticocortico "u-fibres" that connect the ventral premotor cortex with the posterior part of inferior frontal cortex (Broca's area) and the primary motor representations of the articulators. The idea that these small local connections are disrupted is consistent with reports that in PWS the timing and sequence of activation is abnormal between the areas involved in planning speech and the areas involved in executing speech (Salmelin, Schnitzler, Schmitz, \& Freund, 2000). The ventral sensorimotor region also contains fibres that originate from the projection neurons from primary motor cortex to the pons carried in the corticobulbar tract. Disruption in this tract could be related to the speech dysfluency characteristic of stuttered speech. Our tractography analysis aimed to specifically evaluate the integrity of the arcuate fasciculus and corticobulbar tracts in PWS and revealed anomalies in both pathways (see below for discussion).

There are other similarities between the findings of the current tractbased spatial statistics analysis and those of previous studies using this method. In the study of right-handed boys who stutter (Chang et al., 2008), two peaks in the left superior longitudinal fasciculus (-48 -27 25 and -40 -51 40) were described with reduced FA in children who stutter relative to fluent controls that are very closely located to the peaks identified in the current study. Here (see Table 1), these peaks were described as left SLF (mid portion at -37-25 30) and left angular gyrus (-42 -51 43). This latter peak also showed a significant positive correlation with SSI-3 in our analysis: the higher the score on the SSI-3, the lower the FA in this location in PWS. A study of right-handed male PWS (Cykowski et al., 2010) revealed reduced FA in an extensive cluster in the body of the corpus callosum. Consistent with this previous finding, we also report 
reduced FA and increased radial diffusivity in PWS in the posterior portion of the body of the corpus callosum (see Figure 1).

\subsection{Interpreting reduced white matter integrity}

The integrity of white matter is quantified by the ratio of longitudinal to radial diffusion (FA) in the tensor model that is fit to water diffusion measurements made in each voxel of the diffusion image. Areas showing lower FA in PWS compared to controls are described as showing reduced white matter intergrity. It is important to note that low FA could reflect differences in several features of the underlying microstructure, such as myelin and axonal membrane integrity, the packing density and caliber of axons (Beaulieu 2009). Consequently, low FA might affect the speed and quality of signal transmission between brain areas and the synchronization of signals across them. The areas of the cerebral white matter showing low FA in PWS were further explored by comparing radial and longitudinal diffusivities in the same regions. In all areas where FA was low in PWS, longitudinal diffusivity was also low. Radial diffusivity was higher in PWS than in controls only in a small number of regions with low FA, notably the body of the corpus callosum, where it is thought to reflect abnormal myelination of the fibers in this region (Cykowski et al., 2010). In areas where longitudinal diffusivity was low but radial diffusivity was not different in PWS relative to controls, we can speculate that it is fiber organization rather than myelin that is disrupted. However, such reverse inference should be applied cautiously.

\subsection{The relevance of finding abnormalities bilaterally}

The growing consensus towards replicable findings in diffusion-tensor imaging studies of developmental stuttering is encouraging and points to the greater sensitivity of this method compared with morphometric analyses of cortical grey matter. Nevertheless, there were also some striking differences between the current study and other findings. The most notable difference was the frequency with which anomalies were observed in white matter tracts in both hemispheres that were in almost symmetrically homologous brain areas. Only a minority of the white matter differences occurred unilaterally. Based on the uncorrected statistical threshold of $\mathrm{p}<0.005$ that was used, a false positive 
result would occur in 1/200 voxels examined. We attempted to mitigate this false positive rate by requiring the clusters that were reported to show significant differences to have at least 10 neighboring voxels that also survived this statistical threshold (this is a stringent extent threshold, particularly as the skeleton is only one millimeter thick, which means that clusters can only extend along the skeleton). Voxels in the hemisphere contralateral to these small clusters were examined bilaterally to search for homologous anomalies. The likelihood of two voxels occurring by chance in homologous regions in both hemispheres is very small. We propose that the presence of these low probability differences bilaterally reflects an early, possibly genetic etiology.

Structural abnormalities that occur bilaterally are typical of developmental disorders, particularly those with a suspected or known genetic etiology (e.g. verbal apraxia, Belton, Salmond, Watkins, Vargha-Khadem, \& Gadian, 2003; specific language impairment, Badcock, Bishop, Hardiman, Barry, \& Watkins, 2012; schizophrenia, Douaud et al., 2007). It seems plausible that the genetic disruption would have effects on similar structures in each hemisphere rather than acting unilaterally. Congenital disruption would, therefore, suggest that these abnormalities are potentially causal factors in the speech disorder rather than occurring as a consequence of it. Myelination of left language areas typically occurs later than other areas, at 35 months of age (Pujol et al., 2006), preceding but close to the typical onset of developmental stuttering. It could be that this late myelination phase further exaggerates the effects of a pre-existing difference in organization of white matter tracts in children who stutter. Reduced white matter integrity in both hemispheres would also explain the lack of successful reorganization of function between hemispheres, which is a typically seen in focal unilateral lesions acquired congenitally or in childhood (Bates, Vicari, \& Trauner, 1999; Vargha-Khadem, Isaacs, Watkins, \& Mishkin, 2000). The possibility that some of the structural differences observed (particularly the unilateral ones) are a consequence of maladaptive functional reorganization cannot be ruled out (Lu et al., 2012). Interesting questions remain about plasticity and recovery during childhood and more frequent recovery in female children (Ingham et al., 2004). There is a pressing need for longitudinal 
studies from early in development to address the mechanisms of recovery from stuttering (Howell, 2010).

\subsection{Implication of cerebral tract differences}

We used targeted analyses of probabilistic tractography to dissect out specific anatomical pathways in individual participants so that the white matter tracts connecting areas involved in motor control and language could be explored further. We were careful to track within white matter rather than into the cortex, where cortical differences (e.g. Cykowski et al., 2007; Foundas et al., 2004) could contribute to the estimate of FA and add variance to connectivity measures. We specifically selected the arcuate fasciculus, corticospinal and corticobulbar tracts and cerebellar peduncles for analysis, as measurements in these tracts are highly reproducible using our standardized methods (see Giorgio et al., 2010) and these are likely candidates for disruption in developmental stuttering.

The arcuate fasciculus is one of several major tracts comprising the superior longitudinal fasciculus (see above). The standardized tractography analysis successfully dissected the arcuate fibers by placing a seed mask in the middle of this white matter bundle and retaining the tracts that connected it to two target masks located anteriorly in premotor white matter and caudally in posterior temporal white matter. Although the volume of these tracts did not differ between PWS and CON in either hemisphere, the integrity of the tracts (as indexed by FA) was reduced in PWS. The reduced integrity in PWS was seen in both hemispheres. However, in the left hemisphere such a disruption would likely impair the communication between auditory areas in the posterior temporal cortex and motor areas in the frontal lobes, and these interactions are necessary for fluent speech production (Rauschecker \& Scott, 2009). A similar level of anomaly in the right hemisphere might mean that any recovery of function mediated by reorganization to the right hemisphere homologues of speech areas would be suboptimal too.

Dissecting pathways that contribute to speech-motor function separately from those with more general motor function has potential to clarify the picture of white matter abnormalities in PWS. The corticobulbar tract carries the axons of upper motor neurons from the ventral part of the motor cortex via the genu of 
the internal capsule to the pons where it innervates the brainstem nuclei of the cranial nerves that supply the muscles involved in articulation and phonation. The integrity of this tract from the most dorsal part of the capsule, where fibers funnel in from the lateral convexity and travel via the posterior limb to the pons, was examined. Data from this tract were compared to data from the same portion of the corticospinal tract that runs in the posterior limb alongside the corticobulbar tract. The connectivity of the left corticobulbar tract was weaker in PWS relative to controls and relative to its right hemisphere counterpart. Connectivity strength was quantified using the number of streamlines in the tract, which is a difficult metric to interpret (see Jones, Knösche, \& Turner, 2012). Nevertheless, the difference between hemispheres in the PWS group and between the PWS and CON groups is strongly suggestive of a selective impairment in the PWS. As only the left tract was affected, this might reflect persistent dysfunction in the left-lateralized motor control of speech. There was, however, an unexpected inverse relationship between stuttering severity and reduced connectivity as measured by number of streamlines: those with greatest dysfluency had the highest number of streamlines in the left corticobulbar tract. One possible interpretation of this apparently contradictory result is that those PWS with the weakest connectivity in the left corticobulbar tract (i.e. the greatest structural abnormality) have been forced to reorganize speech motor function to, or recruit involvement of the right tract. Hypothetically, successful reorganization would have resulted in greater fluency and, therefore, lower scores on the SSI-3; reorganization would not have occurred in those individuals with milder abnormality of the left tract. This hypothesis predicts a negative relationship between stuttering severity and the connectivity of the right corticobulbar tract (more streamlines, the lower the severity rating). Our analysis did not support this hypothesis, however, as the relationship was positive and not quite significant.

The corticobulbar and corticospinal tracts continue from the internal capsule to the brainstem or spinal cord via the crus cerebri in the cerebral peduncles. The tract-based spatial statistics analysis revealed bilateral reductions in FA in the cerebral peduncles (see Table 1 and Figure 1). Acquired lesions in this area affecting the pons and midbrain can cause neurogenic 
stuttering (e.g. Balasubramanian, Max, Van Borsel, Rayca, \& Richardson, 2003; Doi et al., 2003). Together our results for the cerebral peduncles and the posterior limb of the internal capsule strongly implicate impairment in the descending corticobulbar or cortico-pontine pathways in developmental stuttering.

\subsection{Implications of cerebellar differences in PWS}

Functional imaging studies have reported overactivity of the cerebellar vermis in developmental stuttering during speech production (see Brown et al., 2005). It has been proposed that the cerebellum integrates sensory afference and motor efference to build forward models that predict the consequences of motor acts (Miall, Weir, Wolpert, \& Stein, 1993; Wolpert, Miall, \& Kawato, 1998). As such, abnormal activity in the cerebellum in PWS may be indicative of suboptimal internal models during speech (Max et al., 2004). The integrity of the major white matter fiber bundles, namely the cerebellar peduncles connecting the cerebellum to the rest of the brain, as well as the white matter within the cerebellar lobes and vermis were investigated. There were no significant differences in the white matter in body of the cerebellum but all three peduncles showed reduced integrity bilaterally in PWS. The cerebellum receives the majority of its afferent input through the middle cerebellar peduncle, the majority of which originates in the contralateral pons. The pons in turn receives direct and collateral input from the majority of the cortical mantle including upper motor neurons conveying motor commands via the corticobulbar and corticospinal tracts. Copies of these signals are conveyed to the posterior lobe of the cerebellum along with sensory input from the periphery via proprioceptive and kinesthetic inputs carried mainly in the inferior cerebellar peduncle. Information from the cerebellum is then fed back to the contralateral motor cortex from the cerebellar nuclei via the superior cerebellar peduncle, through the ventrolateral nucleus of the thalamus. One theoretical function of these cerebellar loops is that the feedback from the cerebellum allows the system to adjust ongoing movements and correct errors. It is unclear in stuttering whether there is a primary disruption of the signals to the cerebellum from the motor cortex about the motor commands or the inputs fed back from the periphery or whether it is the integration of these signals that is impaired (Max et al., 2004). 
In any case, the current analysis shows that all possible pathways via the three peduncles have abnormal microstructure that might reduce the quality or subtly affect the timing of signals in these important feedback loops. In particular, the timing of planning and execution signals is thought to contribute to stuttered speech (e.g. EXPLAN model, Howell, 2004).

\subsection{The effects of sex, handedness and age}

The potential effects of sex and handedness on neuroanatomical development are not yet well understood. Instead of limiting our sample to a specific gender or handedness, we attempted to evaluate their contribution to the differences we observed in white matter. The small numbers of subgroups of left-handed males and left- or right-handed females who stutter in our study meant it was not possible to examine the interaction between sex and handedness. Also, data was available in only two left-handed controls making between group comparisons impossible. Nevertheless, our exploratory analysis revealed an interesting difference between left- and right-handers who stutter in the asymmetry of the cortico-spinal tract. The right-handed PWS showed a rightward asymmetry whereas the left-handed PWS showed symmetry similar to the pattern seen for this tract in controls. The structural brain differences between males and females who stutter and the effect of handedness within each group requires further evaluation with larger numbers.

The age range of our PWS and control groups was well matched. Within the PWS there was a significant prediction of white matter integrity in the inferior cerebellar peduncles by age. This relationship was not observed in the controls (see Figure 5C). As age and years of stuttering are strongly related in persistent stuttering, this increase in white matter integrity with age might reflect experience-dependent plasticity that is unique to the stuttering group. Longitudinal studies are ideal for testing for this effect further.

\section{Conclusion}

A comprehensive analysis of a sizeable sample of PWS replicated previously reported reductions in white matter integrity and extended them using a more targeted approach to tracts of interest. The general picture remains complicated 
but several interesting associations between white matter differences, speech dysfluency, handedness, and age warrant further study.

\section{Acknowledgements}

We wish to thank all our participants for their continued contributions to our research. We also thank Dr. Patricia Gough for her assistance with data collection. We thank Dr. Alina Jurcoane for useful discussions of tractography and our colleagues at FMRIB, particularly Drs. Saad Jbabdi, Stamatios Sotiropoulos, and Prof. Steve Smith for analysis support. We thank Ned Jenkinson for useful comments on the manuscript and anatomy. The study was funded in part by a Medical Research Council (UK) grant to KEW (G0400298). This work was completed through the support of the University of Oxford Clarendon Fund Scholarship (EC).

\section{References}

Alm, P. A. (2004). Stuttering and the basal ganglia circuits: a critical review of possible relations. Journal of communication disorders, 37(4), 325-369.

Andrews, G., \& Harris, M. (1964). The Syndrome of Stuttering. London, UK: William Heinemann Medical Books.

Badcock, N. A., Bishop, D. V. M., Hardiman, M. J., Barry, J. G., \& Watkins, K. E. (2012). Co-localisation of abnormal brain structure and function in specific language impairment. Brain and language, 120(3), 310-20.

Balasubramanian, V., Max, L., Van Borsel, J., Rayca, K. O., \& Richardson, D. (2003). Acquired stuttering following right frontal and bilateral pontine lesion: a case study. Brain Cogn, 53(2), 185-189.

Bates, E., Vicari, S., \& Trauner, D. (1999). Neural mediation of language development: perspectives from lesion studies of infants and children. In $\mathrm{H}$. Tager-Flusberg (Ed.), Neurodevelopmental Disorders (pp. 533-581). Cambridge, MA: MIT Press.

Beal, D. S., Gracco, V. L., Lafaille, S. J., \& De Nil, L. F. (2007). Voxel-based morphometry of auditory and speech-related cortex in stutterers. NeuroReport, 18(12), 1257-1260. 
Beaulieu, C. (2009). The biological basis of diffusion anisotropy. In H. JohansenBerg and T. Behrens (Eds.), Diffusion MRI: from quantitative measurement to in-vivo neuroanatomy, (pp 106-123).London, UK: Academic Press.

Behrens, T. E. J., Berg, H. J., Jbabdi, S., Rushworth, M. F. S., \& Woolrich, M. W. (2007). Probabilistic diffusion tractography with multiple fibre orientations: What can we gain? NeuroImage, 34(1), 144-55.

Behrens, T. E. J., Woolrich, M. W., Jenkinson, M., Johansen-Berg, H., Nunes, R. G., Clare, S., Matthews, P. M., et al. (2003). Characterization and propagation of uncertainty in diffusion-weighted MR imaging. Magnetic resonance in medicine: official journal of the Society of Magnetic Resonance in Medicine / Society of Magnetic Resonance in Medicine, 50(5), 1077-88.

Belton, E., Salmond, C. H., Watkins, K. E., Vargha-Khadem, F., \& Gadian, D. G. (2003). Bilateral brain abnormalities associated with dominantly inherited verbal and orofacial dyspraxia. Hum Brain Mapp, 18(3), 194-200.

Bonekamp, D., Nagae, L. M., Degaonkar, M., Matson, M., Abdalla, W. M. A., Barker, P. B., Mori, S., et al. (2007). Diffusion tensor imaging in children and adolescents: reproducibility, hemispheric, and age-related differences. NeuroImage, 34(2), 733-42.

Brown, S., Ingham, R. J., Ingham, J. C., Laird, A. R., \& Fox, P. T. (2005). Stuttered and fluent speech production: An ALE meta-analysis of functional neuroimaging studies. Human Brain Mapping, 25(1), 105-117.

Catani, M., Howard, R. J., Pajevic, S., \& Jones, D. K. (2002). Virtual in Vivo Interactive Dissection of White Matter Fasciculi in the Human Brain. NeuroImage, 17(1), 77-94.

Catani, M., Jones, D. K., \& Ffytche, D. H. (2005). Perisylvian language networks of the human brain. Annals of neurology, 57(1), 8-16.

Chang, S.-E., Erickson, K. I., Ambrose, N. G., Hasegawa-Johnson, M. A., \& Ludlow, C. L. (2008). Brain anatomy differences in childhood stuttering. NeuroImage, 39(3), 1333-1344.

Chang, S.-E., Horwitz, B., Ostuni, J., Reynolds, R., \& Ludlow, C. L. (2011). Evidence of Left Inferior Frontal-Premotor Structural and Functional Connectivity Deficits in Adults Who Stutter. Cerebral cortex (New York, N.Y.: 1991), 21(11), bhr028-.

Chang, S.-E., Kenney, M. K., Loucks, T. M. J., \& Ludlow, C. L. (2009). Brain activation abnormalities during speech and non-speech in stuttering speakers. NeuroImage, 46(1), 201-212. 
Choo, A. L., Chang, S.-E., Zengin-Bolatkale, H., Ambrose, N. G., \& Loucks, T. M. (2012). Corpus callosum morphology in children who stutter. Journal of communication disorders, 45(4), 279-89.

Choo, A. L., Kraft, S. J., Olivero, W., Ambrose, N. G., Sharma, H., Chang, S.-E., \& Loucks, T. M. (2011). Corpus callosum differences associated with persistent stuttering in adults. Journal of communication disorders, 44(4), 470-7.

Cykowski, M. D., Fox, P. T., Ingham, R. J., Ingham, J. C., \& Robin, D. A. (2010). A study of the reproducibility and etiology of diffusion anisotropy differences in developmental stuttering: a potential role for impaired myelination. NeuroImage, 52(4), 1495-1504.

Cykowski, M. D., Kochunov, P. V., Ingham, R. J., Ingham, J. C., Mangin, J.-F. F., Rivière, D., Lancaster, J. L., et al. (2007). Perisylvian Sulcal Morphology and Cerebral Asymmetry Patterns in Adults Who Stutter. Cereb Cortex, 18(3), 571-583.

De Nil, L. F., Kroll, R. M., Kapur, S., \& Houle, S. (2000). A positron emission tomography study of silent and oral single word reading in stuttering and nonstuttering adults. J Speech Lang Hear Res, 43(4), 1038-1053.

Diedrichsen, J. (2006). A spatially unbiased atlas template of the human cerebellum. NeuroImage, 33(1), 127-38.

Doi, M., Nakayasu, H., Soda, T., Shimoda, K., Ito, A., \& Nakashima, K. (2003). Brainstem infarction presenting with neurogenic stuttering. Intern Med, 42(9), 884-887.

Douaud, G., Smith, S., Jenkinson, M., Behrens, T., Johansen-Berg, H., Vickers, J., James, S., et al. (2007). Anatomically related grey and white matter abnormalities in adolescent-onset schizophrenia. Brain, 130(Pt 9), 23752386.

Foundas, A. L., Bollich, A. M., Corey, D. M., Hurley, M., \& Heilman, K. M. (2001). Anomalous anatomy of speech-language areas in adults with persistent developmental stuttering. Neurology, 57(2), 207-215.

Foundas, A. L., Bollich, A. M., Feldman, J., Corey, D. M., Hurley, M., Lemen, L. C., \& Heilman, K. M. (2004). Aberrant auditory processing and atypical planum temporale in developmental stuttering. Neurology, 63(9), 1640-1646.

Foundas, A. L., Corey, D. M., Angeles, V., Bollich, A. M., Crabtree-Hartman, E., \& Heilman, K. M. (2003). Atypical cerebral laterality in adults with persistent developmental stuttering. Neurology, 61(10), 1378-1385.

Geschwind, N. (1975). The apraxias: neural mechanisms of disorders of learned movement. American scientist, 63(2), 188-95. 
Giorgio, A., Watkins, K. E., Chadwick, M., James, S., Winmill, L., Douaud, G., De Stefano, N., et al. (2010). Longitudinal changes in grey and white matter during adolescence. NeuroImage, 49(1), 94-103.

Giraud, A.-L. L., Neumann, K., Bachoud-Levi, A.-C. C., von Gudenberg, A. W., Euler, H. A., Lanfermann, H., \& Preibisch, C. (2008). Severity of dysfluency correlates with basal ganglia activity in persistent developmental stuttering. Brain Lang, 104(2), 190-199.

Greve, D. N., \& Fischl, B. (2009). Accurate and robust brain image alignment using boundary-based registration. NeuroImage, 48(1), 63-72.

Hillis, A. E. (2007). Aphasia: progress in the last quarter of a century. Neurology, $69(2), 200-13$.

Howell, P. (2004). Assessment of some contemporary theories of stuttering that apply to spontaneous speech. Contemporary Issues in Communicative Sciences and Disorders, 39, 122-139.

Howell, P. (2010). Behavioral effects arising from the neural substrates for atypical planning and execution of word production in stuttering. Experimental neurology, 225(1), 55-9.

Ingham, R. J., Fox, P. T., Ingham, J. C., Xiong, J., Zamarripa, F., Hardies, L. J., \& Lancaster, J. L. (2004). Brain correlates of stuttering and syllable production: gender comparison and replication.J Speech Lang Hear Res, $47(2), 321-341$.

Jancke, L., Hanggi, J., \& Steinmetz, H. (2004). Morphological brain differences between adult stutterers and non-stutterers. BMC Neurology, 4, 23.

Jones, D. K., Knösche, T. R., \& Turner, R. (2012). White Matter Integrity, Fiber Count, and Other Fallacies: The Do's and Don'ts of Diffusion MRI. NeuroImage, null(null).

Kell, C. A., Neumann, K., von Kriegstein, K., Posenenske, C., von Gudenberg, A. W., Euler, H., \& Giraud, A.-L. L. (2009). How the brain repairs stuttering. Brain, 132(Pt 10), 2747-2760.

Klein, J. C., Lorenz, B., Kang, J.-S., Baudrexel, S., Seifried, C., van de Loo, S., Steinmetz, H., et al. (2011). Diffusion tensor imaging of white matter involvement in essential tremor. Human brain mapping, 32(6), 896-904.

Loucks, T., Kraft, S. J., Choo, A. L., Sharma, H., \& Ambrose, N. G. (2011). Functional brain activation differences in stuttering identified with a rapid fMRI sequence. Journal of Fluency Disorders. 
Lu, C., Chen, C., Ning, N., Ding, G., Guo, T., Peng, D., Yang, Y., et al. (2010). The neural substrates for atypical planning and execution of word production in stuttering. Experimental Neurology, 221(1), 146-156.

Lu, C., Chen, C., Peng, D., You, W., Zhang, X., Ding, G., Deng, X., et al. (2012). Neural anomaly and reorganization in speakers who stutter: A short-term intervention study. Neurology, 79(7), 625-32.

Ludlow, C. L., \& Loucks, T. (2003). Stuttering: a dynamic motor control disorder. Journal of Fluency Disorders, 28, 273-295.

Max, L., Guenther, F. H., Gracco, V. L., Ghosh, S., S., \& Wallace, M. E. (2004). Unstable or Insufficiently Activated Internal Models and Feedback-Biased Motor Control as Sources of Dysfluency: A Theoretical M. Contemporary Issues in Communication Science and Disorders, 31, 105-122.

Miall, R. C., Weir, D. J., Wolpert, D. M., \& Stein, J. F. (1993). Is the cerebellum a smith predictor? Journal of motor behavior, 25(3), 203-16.

Mock, J. R., Zadina, J. N., Corey, D. M., Cohen, J. D., Lemen, L. C., \& Foundas, A. L. (2012). Atypical brain torque in boys with developmental stuttering. Developmental neuropsychology, 37(5), 434-52.

Månsson, H. (2000). Childhood stuttering. Journal of Fluency Disorders, 25(1), 4757.

Neilson, M. D., \& Neilson, P. D. (1987). Speech motor control and stuttering: A computational model of adaptive sensory-motor processing. Speech Communication, 6(4), 325-333.

Petrides, M., \& Pandya, D. N. (2002). Association pathways of the prefrontal cortex and functional observations. In D. T. Stuss \& R. T. Knight (Eds.), Principles of frontal lobe function. (pp. 31-50). Oxford University Press.

Petrides, M., \& Pandya, D. N. (2009). Distinct parietal and temporal pathways to the homologues of Broca's area in the monkey. PLoS biology, 7(8), e1000170.

Powell, J. L., Kemp, G. J., Roberts, N., \& García-Finaña, M. (2012). Sulcal morphology and volume of Broca's area linked to handedness and sex. Brain and language, 121(3), 206-18.

Pujol, J., Soriano-Mas, C., Ortiz, H., Sebastián-Gallés, N., Losilla, J. M., \& Deus, J. (2006). Myelination of language-related areas in the developing brain. Neurology, 66(3), 339-43.

Rauschecker, J. P., \& Scott, S. K. (2009). Maps and streams in the auditory cortex: nonhuman primates illuminate human speech processing. Nature neuroscience, 12(6), 718-24. 
Riley, G. D. (1994). SSI-3 Stuttering Severity Instrument for Children and Adults (Third.). Austin, TX: Pro-Ed.

Salmelin, R., Schnitzler, A., Schmitz, F., \& Freund, H. J. (2000). Single word reading in developmental stutterers and fluent speakers. Brain, 123 ( Pt 6, 11841202.

Smith, S. M., Jenkinson, M., Johansen-Berg, H., Rueckert, D., Nichols, T. E., Mackay, C. E., Watkins, K. E., et al. (2006). Tract-based spatial statistics: voxelwise analysis of multi-subject diffusion data. NeuroImage, 31(4), 1487-1505.

Sommer, M., Koch, M. A., Paulus, W., Weiller, C., \& Büchel, C. (2002). Disconnection of speech-relevant brain areas in persistent developmental stuttering. Lance, 360(9330), 380-383.

Strub, R. L., Black, F. W., \& Naeser, M. A. (1987). Anomalous dominance in sibling stutterers: evidence from CT scan asymmetries, dichotic listening, neuropsychological testing, and handedness. Brain Lang, 30(2), 338-350.

Travis, L. E. (1978). The cerebral dominance theory of stuttering: 1931--1978. The Journal of speech and hearing disorders, 43(3), 278-81.

Vargha-Khadem, F., Isaacs, E., Watkins, K. E., \& Mishkin, M. (2000, November 7). Ontogenetic specialisation of hemispheric function. W.B Suanders.

Watkins, K. E., Smith, S. S. M., Davis, S., \& Howell, P. (2008). Structural and functional abnormalities of the motor system in developmental stuttering. Brain: a journal of neurology, 131(Pt 1), 50-9.

Wolpert, D. M., Miall, R. C., \& Kawato, M. (1998). Internal models in the cerebellum. Trends in Cognitive Sciences, 2(9), 338-347.

Xuan, Y., Meng, C., Yang, Y., Zhu, C., Wang, L., Yan, Q., Lin, C., et al. (2012). Restingstate brain activity in adult males who stutter. (A. Rodriguez-Fornells, Ed.)PloS one, 7(1), e30570.

Yairi, E., \& Ambrose, N. G. (1999). Early childhood stuttering I: persistency and recovery rates. J Speech Lang Hear Res, 42(5), 1097-1112. 
Table 1: White matter regions showing significant differences between people who stutter and controls in the tract-based spatial statistics analysis

\begin{tabular}{|c|c|c|c|c|c|c|}
\hline White Matter Region & $\mathbf{X}$ & $\mathbf{Y}$ & $\mathbf{Z}$ & $\begin{array}{c}p \text { FA } \\
\text { PWS }< \\
\text { CON }\end{array}$ & \# voxels & $\begin{array}{c}p \text { RD } \\
\text { PWS > } \\
\text { CON }\end{array}$ \\
\hline L superior frontal gyrus & -10 & 50 & 34 & 0.0005 & 16 & \\
\hline R superior frontal gyrus & 10 & 50 & 34 & 0.0005 & $4^{*}$ & 0.011 \\
\hline \multirow[t]{2}{*}{$\begin{array}{r}\text { L inferior frontal gyrus } \\
\text { (anterior) }\end{array}$} & -31 & 38 & 5 & 0.0013 & 11 & \\
\hline & -40 & 35 & 0 & 0.0010 & 10 & \\
\hline \multirow[t]{2}{*}{$\begin{array}{r}\text { R inferior frontal gyrus } \\
\text { (anterior) }\end{array}$} & 32 & 37 & 7 & 0.0023 & $2^{*}$ & \\
\hline & 39 & 33 & -4 & 0.0008 & 8 & \\
\hline L middle frontal gyrus & -35 & 11 & 43 & 0.0006 & 10 & \\
\hline L ventral premotor & -41 & 8 & 16 & 0.0012 & 11 & \\
\hline R ventral premotor & 38 & 8 & 19 & 0.0041 & $3^{*}$ & \\
\hline L anterior corona radiata & -27 & 4 & 30 & 0.0013 & 11 & \\
\hline L cerebral peduncle & -8 & -15 & -19 & 0.0003 & 11 & 0.016 \\
\hline R cerebral peduncle & 9 & -11 & -15 & 0.0036 & $3^{*}$ & \\
\hline $\begin{array}{r}\text { Body corpus callosum } \\
\text { (posterior part) }\end{array}$ & 5 & -24 & 24 & 0.0005 & 11 & 0.022 \\
\hline $\begin{array}{r}\text { L superior longitudinal } \\
\text { fasciculus (mid) }\end{array}$ & -37 & -25 & 30 & 0.0011 & 27 & \\
\hline R angular gyrus & 41 & -52 & 42 & 0.0001 & 18 & 0.010 \\
\hline L angular gyrus & -42 & -51 & 43 & 0.0005 & $5^{*}$ & 0.022 \\
\hline $\begin{array}{r}\mathrm{L} \text { middle temporal gyrus } \\
\text { (posterior) }\end{array}$ & -42 & -59 & 11 & 0.0001 & 12 & \\
\hline R fusiform gyrus (mid) & 37 & -45 & -12 & 0.0001 & 25 & $0.001^{\#}$ \\
\hline L forceps major & -23 & -43 & 25 & 0.0005 & 36 & \\
\hline \multirow[t]{2}{*}{$\mathrm{R}$ forceps major } & 32 & -58 & -10 & 0.0004 & 10 & \\
\hline & 29 & -61 & 15 & 0.0003 & 11 & \\
\hline \multirow[t]{2}{*}{ L optic radiation } & -36 & -52 & 6 & 0.0005 & 15 & 0.025 \\
\hline & -29 & -71 & 12 & 0.0007 & 19 & \\
\hline \multirow[t]{2}{*}{$\mathrm{R}$ optic radiation } & 39 & -54 & 3 & 0.0017 & $2^{*}$ & \\
\hline & 25 & -78 & 3 & 0.0003 & 56 & 0.005 \\
\hline R lateral occipital & 37 & -76 & -6 & 0.0001 & 12 & \\
\hline L lateral occipital & -33 & -77 & -1 & 0.0004 & 23 & \\
\hline \multirow[t]{2}{*}{ R ventral occipital } & 15 & -82 & -5 & 0.0001 & 11 & \\
\hline & & & & $\begin{array}{c}p \text { FA } \\
\text { PWS > } \\
\text { CON }\end{array}$ & & $\begin{array}{c}p \text { RD } \\
\text { CON > } \\
\text { PWS }\end{array}$ \\
\hline $\begin{array}{r}\text { R middle frontal gyrus } \\
\text { (anterior) }\end{array}$ & 33 & 37 & 22 & 0.0002 & 22 & 0.025 \\
\hline L middle frontal gyrus (mid) & -32 & 19 & 34 & 0.0003 & 10 & \\
\hline L temporal pole & -37 & 5 & -30 & 0.0003 & 13 & \\
\hline L dorsal precentral gyrus & -15 & -20 & 58 & 0.0006 & 25 & $0.0025^{+}$ \\
\hline R dorsal precentral gyrus & 15 & -17 & 59 & 0.0018 & $4^{*}$ & $0.0008^{+}$ \\
\hline
\end{tabular}

${ }^{*}$ Peaks with fewer than 10 voxels bilateral to white matter regions passing threshold

+ PWS $>$ CON in RD where PWS $>$ CON in FA

\# $\mathrm{PWS}<\mathrm{CON}$ in RD where PWS $<\mathrm{CON}$ in FA 


\section{Figure Captions}

Figure 1. Tract-based spatial statistics analysis of white matter. Blue areas indicate regions in which PWS had significantly reduced FA relative to CON. The map of the $p$-value was thresholded at $p<0.005$ and the remaining voxels thickened for visualization purposes. The underlying brain image is the mean FA across all participants with the white matter skeleton overlaid in green. R, right; $\mathrm{L}$, left. A. Coronal slice at $34 \mathrm{~mm}$ in front of the anterior commissure. Anterior inferior frontal white matter areas are encircled in red. B. Axial slice at $16 \mathrm{~mm}$ below the bicommissural plane. Cerebral peduncles are encircled in red. C. Coronal slice at $8 \mathrm{~mm}$ in front of the anterior commissure. White matter areas underlying the ventral premotor cortex are encircled in red. D. Sagittal slice through the right hemisphere at $5 \mathrm{~mm}$ from the midline. The posterior corpus callosum is encircled in red. E. Graphs showing individual data for FA in the white matter underlying left and right ventral premotor cortex. Blue circles male PWS; blue triangles - female PWS; red circles - male CON; red triangles female CON. Filled - left handers; unfilled - right handers. The horizontal line indicates the group mean.

Figure 2. Probabilistic tractography of arcuate fasciculus and corticospinal tracts. Underlying brain image is the MNI152 template T1-weighted image in standard space. Blue areas are the average of the thresholded tracts across all participants after running probabilistic tractography between the seed masks (red) and the target masks (pink). Termination and exclusion masks (see text for details) are also shown (yellow areas). A - anterior; P - posterior; L - left; R Right. Bars on graphs show means for groups of PWS (blue) and CON (red). Error bars - standard error of the mean. * - significant difference. A. Arcuate fasciculus shown on sagittal slice through the left hemisphere $36 \mathrm{~mm}$ from the midline. Significantly reduced FA bilaterally in PWS relative to CON. B. Corticospinal tract shown on coronal slice at $18 \mathrm{~mm}$ posterior to the anterior commissure. FA is significantly lower in the left hemisphere compared to the right in PWS. 
Figure 3. Probabilistic tractography in the posterior limb of the internal capsule. Underlying brain image is a diffusion image showing the principal diffusion direction at each voxel modulated by the underlying FA (bright areas high FA). Red - diffusion in the left-right axis; green - diffusion in the anterior-posterior axis; blue - diffusion in the superior-inferior axis. Squares outlined show the voxels selected for the target masks in probabilistic tracking of the corticobulbar (CBT; yellow) and corticospinal (CST; red) tracts. L - left; R - Right. Bars on graphs show mean number of streamlines in each tract for groups of PWS (blue) and CON (red). Error bars - standard error of the mean. * - significant difference.

Figure 4. Cerebellar peduncle region-of-interest analysis. Left - underlying brain images are slices through a diffusion image in a single participant (see legend to Figure 3 for details). The superior cerebellar peduncle (SCP) masks are shown in pink, the middle cerebellar peduncle (MCP) masks are shown in light blue and the inferior cerebellar peduncle (ICP) masks are shown in red. L - left; R - Right; A - anterior; P - posterior. Bars on graphs show mean FA for each region-ofinterest in each peduncle for groups of PWS (blue) and CON (red). Error bars standard error of the mean. * - significant difference.

Figure 5. The relationship between stuttering severity, handedness and age and white matter differences in PWS. A. The relationship between FA in the white matter underlying the left angular gyrus and SSI-3 is shown for PWS in blue (line - regression line) and the scatterplot of data in CON in red (line - mean). B. The difference between left- and right-handed PWS in the FA in the left (dark blue) and right (light blue) CST. Error bars - standard error of the mean. * significant difference. C. The relationship between age and FA in the inferior cerebellar peduncle in PWS (blue) and CON (red). Lines are regression lines. 